\title{
CAUSAS DE MORTALIDAD EMBRIONARIA EN LA INCUBACION NATURAL Y ARTIFICIAL DE HUEVOS DE PATA CRIOLLA (Cairina moschata doméstica L.)
}

\author{
CAUSES OF EMBRYONIC MORTALITY IN THE NATURAL AND ARTIFICIAL \\ INCUBATION OF EGGS PATA CRIOLLA (Cairina moschata doméstica L.)
}

\author{
${ }^{1}$ Hilario Hildebrando Ortiz Chavez y ${ }^{2}$ Marcial Estanislao Cumpa Gavidia
}

\begin{abstract}
Resumen
El objetivo del presente estudio fue determinar las causas de mortalidad embrionaria, tanto en incubación natural, así como en incubación artificial de los huevos de pata Criolla. Para ello, se incubaron en total 900 huevos, de los cuales 778 fueron fértiles. Los resultados mostraron que el almacenamiento de los huevos en el nido hasta por tres semanas permitió una mayor viabilidad de los embriones, en comparación con el almacenamiento en sala para incubación artificial. Las causas principales de mortalidad embrionaria, tanto en incubación natural como en la artificial son consecuencia de la contaminación de los huevos, así como de las malposiciones y malformaciones embrionarias. La malposición embrionaria estuvo relacionada con la mortalidad embrionaria, sobre todo en el último tercio del proceso de incubación. Hubo mayor porcentaje de malformaciones embrionarias en la incubación artificial y las causas más frecuentes en ambos tipos de incubación fueron las anormalidades viscerales y desordenes circulatorios.
\end{abstract}

Palabras clave: Mortalidad embrionaria, pata Criolla, malposiciones, malformaciones.

\begin{abstract}
The objective of the present study was to determine the causes of embryonic mortality, both natural and artificial incubation of eggs Muscovy ducks. 900 eggs were incubated, of which 778 were fertile. The results showed that storage of eggs up to three weeks in the nest allowed greater viability of embryos, compared with storage in room for artificial incubation. The main causes of embryonic mortality, both natural and artificial incubation are the result of contamination of eggs, and the embryonic malpositions and malformations. Embryo malposition was associated with embryonic mortality, especially in the last third of the incubation process. There was a higher percentage of embryonic malformations in artificial incubation and the most frequent causes for both types of incubation were visceral abnormalities and circulatory disorders.
\end{abstract}

Keys words: Embryonic mortality, Muscovy duck, malpositions, malformations.

\section{Introducción}

El pato Criollo o Muscovy (Cairina moschata doméstica $L$.), es una especie nativa con buenas características productivas; sin embargo aún no se han realizado suficientes investigaciones para mejorar los nacimientos con el uso de la incubación artificial. Christensen et al. (1996) manifiestan que la fertilidad de los huevos incubables, la mortalidad embrionaria y la incubabilidad son los criterios más importantes de la fertilidad de las aves. La pata anida durante 35 días, realiza ella misma todas las labores de incubación, volteando periódicamente los huevos y proporcionando la temperatura y humedad adecuada (Narbaiza, 2005). En la mortalidad embrionaria en pollos, Kalita et al. (2013) encontraron malposiciones
(19.50\%), malformaciones (7.88\%), adhesión $(2.49 \%)$, deshidratación (1.66\%), condiciones patológicas $(27.80 \%)$ y anormalidades no definidas $(40.66 \%)$. En la incubación de las aves, hay al menos dos cimas de mortalidad embrionaria: La primera, se produce al comienzo de la incubación y la segunda, más pronunciada, se presenta antes que el embrión penetre en la cámara de aire, es decir, 2 a 3 días antes de la fecha de eclosión (Christensen, 2001). En la mortalidad embrionaria durante la incubación artificial de los huevos de pata criolla, la última cima es más elevada (Blas, 1978; Rivas, 1981 y Vargas, 1985). La mortalidad embrionaria tardía se atribuye al defecto de almacenamiento, que reduciría la disponibilidad de glucosa utilizada por el embrión

\footnotetext{
${ }^{1}$ Médico Veterinario. Práctica Privada. EPG - UNALM

${ }^{2}$ Departamento Académico de Producción Animal, Facultad de Zootecnia- UNALM, Lima, Perú. E-mail: mcumpa@lamolina.edu.pe
} 
como fuente de energía para su desarrollo, a tal punto que algunos no son capaces de picar la cáscara (Reinhart y Hurnik, 1976). La frecuencia de malposiciones según Romanoff y Romanoff (1972), está relacionada con la mortalidad total. Si el número de embriones muertos se incrementa, especialmente durante la última parte de la incubación, entonces el número de muertos en diversas malposiciones también se incrementa. Kalita et al. (2013), encontraron que las malposiciones registradas fueron: la cabeza dirigida hacia el lado izquierdo, la cabeza dirigida hacia el extremo agudo del huevo y la cabeza entre los muslos; mientras que las malformaciones observadas fueron: pico corto superior, patas anormales y prolapso intestinal. Cuando el almacenamiento es prolongado, la albumina se degrada permitiendo que la yema gire y flote muy cerca de la parte superior del huevo, de modo que el embrión se sitúa cerca a la membrana interior de la cascara exponiéndose a la deshidratación o a contaminación bacteriana que generan mortalidad embrionaria (Alda, 2003). El porcentaje de células vivas fue significativamente mayor en huevos almacenados durante 4 días (81.17\%) en comparación con aquellos almacenados por 14 días (68.18\%); mientras que las células en apoptosis temprana fueron significativamente mayores en huevos almacenados durante 14 días (17.88\%) comparados con los de menor tiempo de almacenamiento (Hamidu et al., 2010). Asimismo, Reis et al. (1997) observaron que a mayor tiempo de almacenamiento disminuye la calidad del albumen asociada a cascaras de huevo delgadas, que resulta en escape rápido de $\mathrm{CO} 2$, desequilibrio acido-base y finalmente la muerte del embrión. Harun et al. (2001) mencionan que al final de la incubación, el fluido sanguíneo calienta las superficies de los huevos, y el relativo incremento en la temperatura de la superficie de los huevos grandes es mayor que la de los pequeños. El procedimiento de comparación de los embriones y determinación de las curvas de mortalidad son indicios claves para dilucidar los problemas de la incubación. Si se tuviera una idea en cuanto al tiempo de la muerte o al desarrollo estructural del pato criollo se podría enfocar más claramente los problemas de la incubación artificial. Por tanto, el presente estudio tiene como objetivos determinar las causas de mortalidad embrionaria tanto en la incubación natural como artificial en huevos de pata Criolla.

\section{Materiales y métodos}

El presente estudio se llevó a cabo en la Unidad Experimental de Avicultura de la Universidad Nacional Agraria La Molina y tuvo una duración de 7 meses. Se utilizaron 18 patas hembras y 4 patos machos reproductores de la raza Criolla para la producción de huevos destinados a incubación natural; mientras que se emplearon 25 hembras y 5 machos para incubación artificial. Los huevos aptos para incubación fueron almacenados, por un lapso de tiempo de 1, 2 y 3 semanas. Los huevos para incubación natural fueron dejados en el nido, previa identificación y pesado, hasta completar el número adecuado para que la pata entre en cloquez, mientras que los huevos colectados para incubación artificial previa identificación fueron pesados $\mathrm{y}$ almacenados. Estos huevos colocados en las bandejas, en posición vertical y con la punta aguda hacia abajo fueron cargados en la incubadora. La temperatura en incubadora fue de $37.5^{\circ} \mathrm{C}$ y en nacedora de $36.7^{\circ} \mathrm{C}$. La humedad relativa durante la incubación estuvo entre 58 a $60 \%$, mientras en nacedora se mantuvo entre 78 a $80 \%$. La ventilación en la incubadora y en la nacedora era forzada por ventiladores. Se realizaban volteos de los huevos cada dos horas hasta el día 33 de incubación en que eran transferidos a la nacedora. Teniendo como eje horizontal la longitud mayor del huevo, estos eran volteados en un ángulo de $90^{\circ}$. La aspersión de los huevos con agua, en la incubación artificial, era diaria a partir del quinto día de incubación hasta la transferencia a la nacedora y se hacía asperjando los huevos con agua a $33^{\circ} \mathrm{C}$. En los mirajes se buscaba detectar los huevos fértiles con embriones vivos o muertos, así como también los huevos infértiles. En el segundo y el tercer miraje se buscaba detectar los huevos con embriones aparentemente muertos, éstos eran abiertos y examinados para confirmar su condición, así como para estimar el momento de la muerte del embrión. La transferencia de los huevos a la nacedora se efectuaba el día correspondiente al último miraje, previa evaluación del peso. Los patos BB y sus cascarones fueron retirados de la nacedora en el día del nacimiento, procediéndose a la identificación y determinación del extremo por el cual salía el patito del cascarón. Los huevos no eclosionados al final del nacimiento fueron examinados para determinar: huevos con cáscara sin picar, huevos con cáscara picada, huevos con cáscara sin picar y membrana interna rasgada, y posición del embrión. El tiempo de desarrollo de los embriones fue dividido en tres períodos: desarrollo temprano (hasta 14 días de incubación), desarrollo medio (15 a 30 días de incubación), desarrollo final (31 a 35 días de incubación). Los huevos fértiles fueron clasificados por tiempo de almacenamiento en huevos de 0 a 7 días, de 8 a 15 días, y de 16 a 21 días de almacenamiento. Se utilizó la prueba de Chi-Cuadrado ( $\chi 2)$ para evaluar la fertilidad, incubabilidad y mortalidad por tipo de incubación, así como para comparar la mortalidad embrionaria por tiempo de almacenamiento en incubación natural y artificial, y para comparar las causas de mortalidad embrionaria por tipo de incubación.

\section{Resultados y discusión}

En la Tabla 1, se presenta el número de incubaciones controladas, huevos incubados, huevos infértiles, huevos fértiles, embriones muertos y patitos nacidos en ambas clases de incubación. De un total de 900 huevos incubados en ambas clases de incubación resultaron ser fértiles 778 con una fertilidad general de $86.45 \%$, fluctuando entre $86.90 \%$ para la incubación natural y $86.04 \%$ para la incubación artificial. 
Las fertilidades de los huevos incubados bajo los dos métodos fueron similares previo a la evaluación experimental por proceder de un mismo lote de reproductores, en cambio la incubabilidad y la viabilidad de los huevos fértiles fueron afectados por el método de incubación. La natalidad bajo el tratamiento de incubación natural fue significativamente mayor $(65.21 \%)$ comparado con la incubación artificial $(23.73 \%)$. La fertilidad general promedio del experimento fue de $86.45 \%$ cifra que es inferior al rango obtenido por Rivas (1981), pero superior a los trabajos realizados por Candela (1975), Pacheco (1976), Gonzales (1976) y Vela (1982).

La mortalidad embrionaria en incubación natural presentó $34.79 \%$ de mortalidad embrionaria; siendo en la incubación artificial, mucho mayor, con $76.27 \%$.

Tabla 1. Porcentajes de las incubaciones controladas, huevos incubados, huevos infértiles, huevos fértiles, embriones muertos y patitos nacidos en ambas clases de incubación.

\begin{tabular}{lccccccccccc}
\hline \multirow{2}{*}{ Incubación } & \multicolumn{2}{c}{ Incubaciones } & \multicolumn{2}{c}{$\begin{array}{c}\text { Huevos } \\
\text { Incubados }\end{array}$} & \multicolumn{2}{c}{$\begin{array}{c}\text { Huevos } \\
\text { Infertiles }\end{array}$} & \multicolumn{2}{c}{ Huevos Fertiles } & \multicolumn{2}{c}{$\begin{array}{c}\text { Embriones } \\
\text { muertos }\end{array}$} & \multicolumn{2}{c}{ Patos BB } \\
\cline { 2 - 13 } & $\mathrm{N}^{\mathrm{o}}$ & $\%$ & $\mathrm{~N}^{\mathrm{o}}$ & $\mathrm{N}^{\mathrm{o}}$ & $\%$ & $\mathrm{~N}^{\mathrm{o}}$ & $\%$ & $\mathrm{~N}^{\mathrm{o}}$ & $\%$ & $\mathrm{~N}^{\mathrm{o}}$ & $\%$ \\
\hline Natural & 21 & 100 & 420 & 55 & 13.10 & 365 & $86.90^{\mathrm{a}}$ & 127 & $34.79^{\mathrm{b}}$ & 238 & $65.21^{\mathrm{a}}$ \\
Artificial & 12 & 100 & 480 & 67 & 13.96 & 413 & $86.04^{\mathrm{a}}$ & 315 & $76.27^{\mathrm{a}}$ & 98 & $23.73^{\mathrm{b}}$ \\
Total & 33 & 100 & 900 & 122 & 13.55 & 778 & 86.45 & 442 & 56.81 & 336 & 43.19 \\
\hline
\end{tabular}

NOTA: Letras diferentes dentro de columnas indican diferencias significativas.

Para el tratamiento de incubación natural, la tabla 2 registra la incubabilidad de los huevos fértiles que es de $65.21 \%$, siendo inferior a $81.6 \%$ reportado por Pacheco (1976) de huevos incubados luego de ser almacenados en el nido durante 1, 2 y 3 semanas, y al 71.9\% de Candela (1975); pero superior a $53.9 \%$ y $58.1 \%$, alcanzados por Gonzales (1976) y Vela (1982) respectivamente. El tratamiento de incubación artificial tuvo un valor promedio de $23.73 \%$ (Tabla 2), este porcentaje es considerablemente bajo con relación a la incubabilidad natural, que llega a niveles que están dentro de un rango de $71.9 \%$ y $91.2 \%$, reportados por Candela (1975) y Pacheco (1976), respectivamente; el mismo es superior a lo hallado por Gonzales (1976) de 10.2\% y Vilchez (1975), que fue de $20.5 \%$ de incubabilidad. A la prueba de Chi Cuadrado $(\chi 2)$, los resultados de la incubabilidad dieron diferencias altamente significativas entre tratamientos a favor de los huevos incubados en forma natural.

Tabla 2. Embriones muertos e incubabilidad, calculado sobre el número total de huevos fértiles en ambas clases de incubación.

\begin{tabular}{cccccc}
\hline \multirow{2}{*}{ Incubación } & $\begin{array}{c}\text { Huevos } \\
\text { fértiles }\end{array}$ & \multicolumn{2}{c}{$\begin{array}{c}\text { Embriones } \\
\text { muertos }\end{array}$} & \multicolumn{2}{c}{ Incubabilidad } \\
\cline { 2 - 6 } & $\mathrm{N}^{\mathrm{o}}$ & $\mathrm{N}^{\mathrm{o}}$ & $\%$ & $\mathrm{~N}^{\mathrm{o}}$ & $\%$ \\
\hline Natural & 365 & 127 & $34.79^{\mathrm{b}}$ & 238 & $65.21^{\mathrm{a}}$ \\
Artificial & 413 & 315 & $76.27^{\mathrm{a}}$ & 98 & $23.73^{\mathrm{b}}$ \\
Total & 778 & 442 & 56.81 & 336 & 43.19 \\
\hline
\end{tabular}

NOTA: Letras diferentes dentro de columnas indican diferencias significativas.

Al comparar la incubabilidad promedio del experimento con las reportadas por autores que trabajaron bajo condiciones artificiales de incubación, se observa una superioridad con respecto a los promedios generales reportados por Vilchez (1975), González (1976), Blas (1978) y Rivas (1981), que fueron de $20.5 \%, 10.2 \%$, $8.8 \%$ y $18.0 \%$, respectivamente.
En la Tabla 3 se aprecia que el tiempo de almacenamiento de los huevos almacenados a temperatura ambiente tanto en sala como en el nido, tiene influencia sobre la mortalidad de los embriones, ya que los huevos almacenados por una, dos y tres semanas dieron porcentajes de $9.86 \%$, $12.05 \%$ y de $12.88 \%$, y $24.21 \%, 25.91 \%$ y $26.15 \%$ para la incubación natural y artificial respectivamente. Los resultados muestran que la mortalidad embrionaria aumenta conforme aumenta el tiempo de almacenamiento. Los análisis estadístico de la prueba de Chi Cuadrado $(\chi 2)$ para ambos tipos de incubación indican que existen diferencias significativas de mortalidad entre la primera y tercera semana de almacenamiento; mas no entre la primera y la segunda semana de almacenamiento. Esto sugiere que los huevos deben ser almacenados en sala por el menor tiempo posible, en este caso por una semana, lo que concuerda con Pacheco (1976) quien encontró que los huevos almacenados en sala en esta forma hasta por dos semanas, presentaban una mortalidad de $18.4 \%$; otras investigaciones realizadas confirman que las mortalidades aumentan a medida que la longitud del periodo de almacenamiento es incrementado, especialmente después de almacenarlo por más de una semana (Hamidu et al., 2010 y Reis et al., 1997) y que, la menor mortalidad en los huevos almacenados por tiempos prolongados depende de la temperatura del cuarto de almacenamiento. Si bien, el huevo de pata por su mayor porcentaje de albumen denso en comparación con otros huevos de aves domésticas, tendría un mayor período de almacenamiento, este lapso no debe excederse de los 7 días.

Con respecto a las temperaturas recomendadas, para el almacenamiento de los huevos durante tres semanas son preferibles las temperaturas bajas de $10^{\circ} \mathrm{C}$ a $15^{\circ} \mathrm{C}$ para lograr una menor mortalidad embrionaria (Reinhart y Hurnik, 1976). 
La mortalidad embrionaria, expresada en número y porcentaje calculada sobre el número total de huevos fértiles para cada método de incubación, se puede apreciar en la tabla 4 y Gráfico 1. La mortalidad difiere entre clases de incubación, la mayor mortalidad se presenta en los huevos incubados artificialmente $(76.27 \%)$ y la menor en los huevos incubados en forma natural (34.79\%).

La distribución de la mortalidad es similar, pero en la etapa de 25 a 30 días la mortalidad embrionaria es mayor en la incubación artificial (6.3\%) que en la natural (2.46\%), acentuándose más en la última etapa comprendida entre el día 31 y el día de la eclosión, en la que la mortalidad en condiciones artificiales es de $57.38 \%$ y en condiciones naturales es de $14.79 \%$ (Tabla 4).

La distribución de la mortalidad embrionaria presenta dos picos: el primero en el tercio inicial de la incubación y el segundo, más elevado, en los días previos a la fecha de nacimiento, cuando el embrión está por nacer; las distribuciones de la mortalidad reportadas como normalmente encontradas en la incubación de las aves domésticas por Landauer (1967) concuerdan con las encontradas en el presente trabajo. Los dos picos de mortalidad obtenidos en la curva de mortalidad embrionaria son similares a lo reportado por Vilchez (1975) y Gonzales (1976) en incubación de patas, y Christensen (2001) en gallinas. El largo período de incubación, mayor que el de otras aves domésticas, así como el hecho de que las máquinas incubadoras están diseñadas para los procesos de incubación de huevos de gallinas y ajustados a los requerimientos de temperatura, humedad relativa, ventilación y volteo de los embriones de pollo influye también en una menor incubabilidad y por ende una mayor mortalidad embrionaria en los huevos de pata Criolla.

La Tabla 5 reporta la distribución de la mortalidad embrionaria por etapas de desarrollo. En la incubación natural se observa que en el periodo de 0 a 14 días el porcentaje de mortalidad embrionaria es de $11.23 \%$, disminuyendo en la etapa de 15 a 30 días que es de $8.77 \%$ y aumenta a $14.79 \%$ en la etapa de los 31 días a la eclosión. En la incubación artificial, se observa que de 0 a 14 días, la mortalidad embrionaria es de $7.26 \%$, de 15 a 30 días es de $11.62 \%$ y se incrementa a $57.39 \%$ en la etapa de los 31 días a la eclosión.

Tabla 3. Número de huevos fértiles, de embriones muertos y porcentaje de mortalidad embrionaria por semana de almacenamiento en ambas clases de incubación.

\begin{tabular}{lllcccc}
\hline Clases de & & \multicolumn{4}{c}{ Semanas de almacenamiento } \\
\cline { 3 - 6 } incubación & & & 1 & 2 & 3 & Total \\
\hline \multirow{3}{*}{ Natural } & Huevos fértiles & $\mathrm{N}^{\mathrm{o}}$ & 129 & 121 & 115 & 365 \\
& Embriones muertos & $\mathrm{N}^{\mathrm{o}}$ & 36 & 44 & 47 & 127 \\
& Mortalidad embrionaria & $\%$ & $9.86^{\mathrm{b}}$ & $12.05^{\mathrm{b}}$ & $12.88^{\mathrm{a}}$ & 34.79 \\
\multirow{3}{*}{ Artificial } & $\mathrm{N}^{\mathrm{o}}$ & 145 & 137 & 131 & 413 \\
& Huevos fértiles. & $\mathrm{N}^{\mathrm{o}}$ & 100 & 107 & 108 & 315 \\
& Embriones muertos & $\%$ & $24.21^{\mathrm{b}}$ & $25.91^{\mathrm{b}}$ & $26.15^{\mathrm{a}}$ & 76.27 \\
\hline
\end{tabular}

NOTA: Letras diferentes dentro de columnas indican diferencias significativas.

Tabla 4. Mortalidad embrionaria por tiempo de incubación, en incubación natural y artificial.

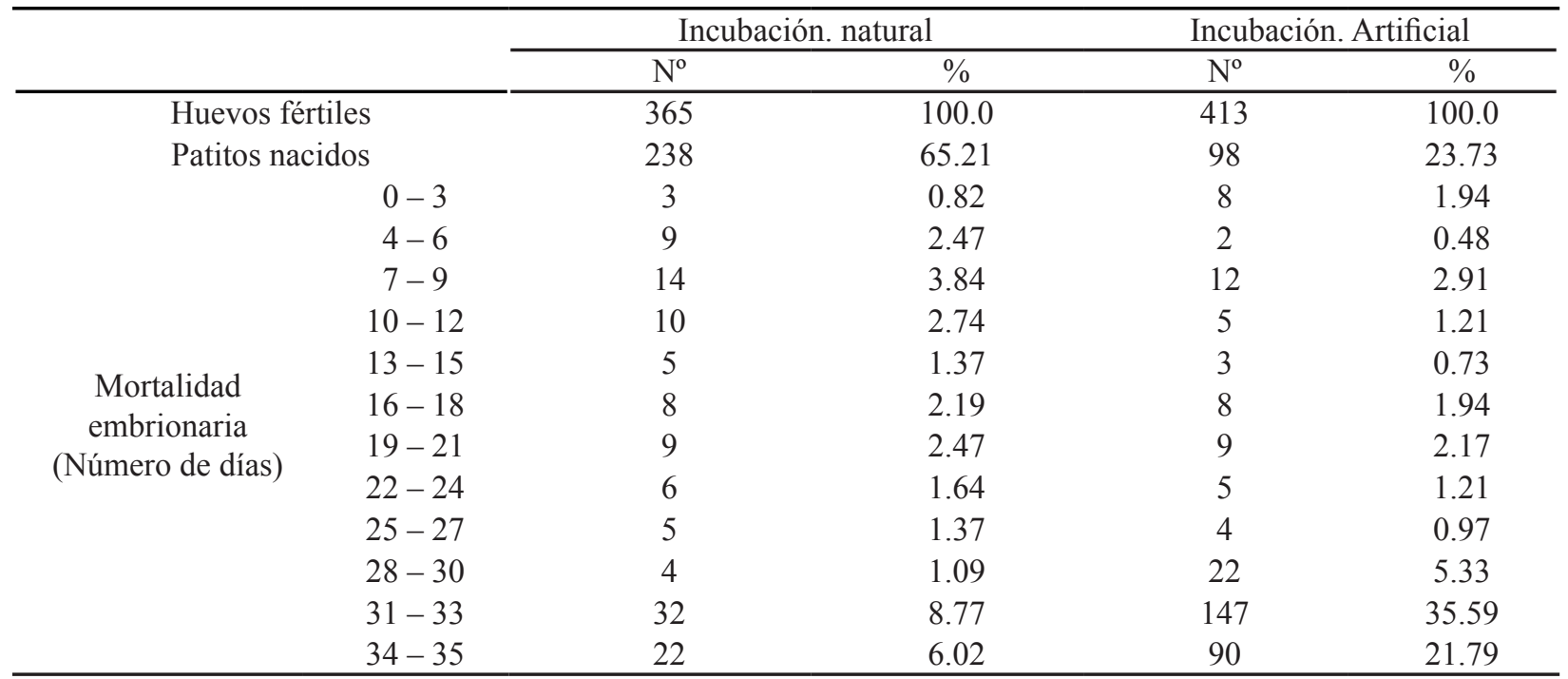


La mortalidad embrionaria en el transcurso de la incubación artificial fue mayor en los dos momentos críticos mencionados, esto es en el transcurso de los siete días, y a nivel de los 30 a 35 días. Esto podría indicar que las temperaturas de incubación en condiciones artificiales durante los siete primeros días influyen desfavorablemente, debilitando a los embriones, de tal modo que para la última etapa de la incubación, no tiene el desarrollo ni la energía suficiente para poder eclosionar, lo que ratifica la importancia de encontrar las temperaturas ideales para este periodo en que se suceden cambios fisiológicos y metabólicos que van a influir en el normal desarrollo del embrión (Landauer, 1967; Reinhart y Hurnik, 1976).

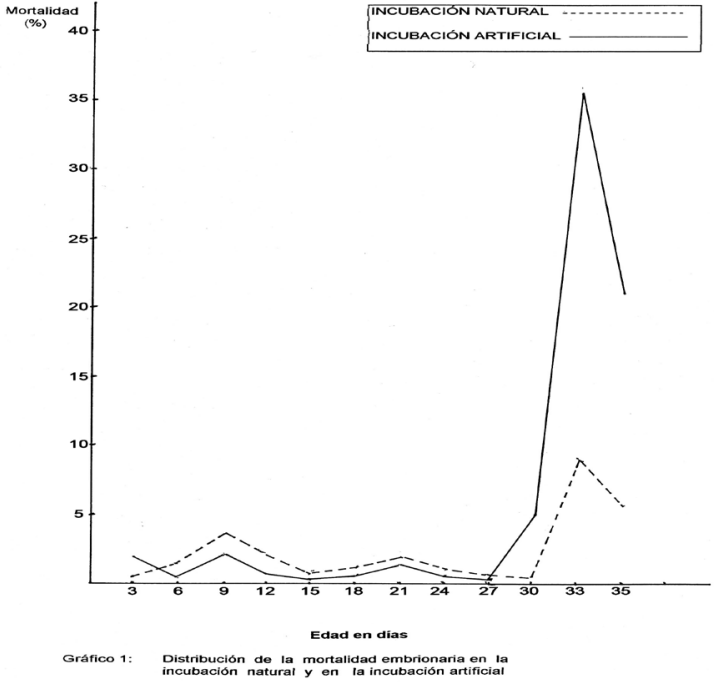

Gráfico 1. Distribución de la mortalidad embrionaria en la incubación natural y en la incubación artificial por tiempo de incubación.

Tabla 5. Número y porcentaje de embriones muertos por etapas de desarrollo, con especificación de los huevos no picados, donde se encuentran los embriones que no rasgaron membrana y embriones que rasgaron membrana, y huevos picados en ambas clases de incubación.

\begin{tabular}{|c|c|c|c|c|c|c|c|c|c|c|}
\hline \multirow{3}{*}{ Incubación } & \multirow{3}{*}{$\begin{array}{l}\text { Etapas de } \\
\text { desarrollo }\end{array}$} & \multicolumn{5}{|c|}{ Huevos no picados } & \multirow[b]{2}{*}{$\begin{array}{c}\text { Mortalidad } \\
\text { total }\end{array}$} & \multirow{2}{*}{\multicolumn{2}{|c|}{$\begin{array}{l}\text { Mortalidad } \\
\text { total }\end{array}$}} & \multirow{3}{*}{$\begin{array}{c}\text { Fertilidad } \\
\text { total }\end{array}$} \\
\hline & & \multicolumn{2}{|c|}{$\begin{array}{c}\text { Embriones que } \\
\text { no rasgaron } \\
\text { membrana }\end{array}$} & \multicolumn{2}{|c|}{$\begin{array}{l}\text { Embriones } \\
\text { que rasgaron } \\
\text { membrana }\end{array}$} & \multirow{2}{*}{$\begin{array}{l}\begin{array}{l}\text { Huevos } \\
\text { picados }\end{array} \\
\mathrm{N}^{\circ} \\
\end{array}$} & & & & \\
\hline & & No & $\%$ & $\mathrm{~N}^{\circ}$ & $\%$ & & $\%$ & $\mathrm{~N}^{\circ}$ & $\%$ & \\
\hline & 0 a 14 días & 41 & 11.23 & -- & -- & -- & -- & & & \\
\hline Natural & 15 a 30 días & 32 & 8.77 & -- & -- & -- & -- & 127 & 34.8 & 365 \\
\hline & 31 a eclosión & 54 & 14.79 & -- & -- & -- & -- & & & \\
\hline & 0 a 14 días & 30 & 7.26 & -- & -- & -- & -- & & & \\
\hline Artificial & 15 a 30 días & 48 & 11.62 & -- & -- & -- & -- & 315 & 76.3 & 413 \\
\hline & 31 a eclosión & 233 & 56.42 & 3 & 0.73 & 1 & 0.24 & & & \\
\hline
\end{tabular}

Tabla 6. Causas de mortalidad embrionaria en ambas clases de incubación

Huevos no picados

Incubación Causas de mortalidad embrionaria

\begin{tabular}{|c|c|c|c|c|c|c|}
\hline & & $\mathrm{N}^{\mathrm{o}}$ & $\%$ & $\mathrm{~N}^{\mathrm{o}}$ & $\%$ & $\mathrm{~N}^{\mathrm{o}}$ \\
\hline \multirow{3}{*}{ Natural } & Huevos contaminados & 73 & 20 & & & \\
\hline & Malposiciones embrionarias & 34 & 9.31 & 127 & 34.79 & 365 \\
\hline & Malformaciones embrionarias & 20 & 5.48 & & & \\
\hline \multirow{3}{*}{ Artificial } & Huevos contaminados & 78 & 18.89 & & & \\
\hline & Malposiciones embrionarias & 170 & 41.16 & 315 & 76.27 & 413 \\
\hline & Malformaciones embrionarias & 67 & 16.22 & & & \\
\hline
\end{tabular}

Es importante dilucidar la incidencia de malposiciones y esto se logro analizando los datos del presente estudio y comparándolos con los que cita Romanoff y Romanoff (1972) quienes reportan que alrededor del 50\% del total de embriones muertos presentaron mal posiciones. Este porcentaje es similar al resultado del presente estudio, que fue de alrededor del $50.47 \%$, siendo para la incubación natural de $9.31 \%$ y para la incubación artificial de $41.16 \%$ (Tabla 6); siendo alto y sometido a trabajo de mejoramiento puede ser minimizado, a través de selección genética y sobre todo en cuanto a manejo nutricional, sanitario y técnicas de incubación. Los 
resultados del presente trabajo coinciden con Romanoff y Romanoff (1972) y Harris (1980), en cuanto a la relación directa que existe entre la mortalidad embrionaria y la frecuencia de malposiciones encontradas en el último tercio del proceso de incubación, por ser en éste el periodo en el cual el pato BB se prepara para nacer. También hubo diferencia con respecto al tipo de malposición entre una clase de incubación y la otra, ya que en la incubación natural (Tabla 7) las más frecuentes fueron: la del tipo IV (pata sobre la cabeza), que Harris (1980) describe como rara y en segundo lugar coincidiendo con Romanoff y Romanoff (1972) que la considera más frecuente, la de tipo VI (cabeza entre las patas), que según San Gabriel (1968), se debe a problemas de contaminación.

Tabla 7. Número y porcentaje de malposiciones embrionarias encontradas en ambas clases de incubación.

\begin{tabular}{|c|c|c|c|c|}
\hline \multirow{3}{*}{$\begin{array}{c}\text { Tipos de } \\
\text { malposición }\end{array}$} & \multicolumn{4}{|c|}{ Mortalidad Embrionaria } \\
\hline & \multicolumn{2}{|c|}{$\begin{array}{c}\text { Incubación } \\
\text { Natural }\end{array}$} & \multirow{2}{*}{$\frac{\text { Incubación }}{\mathrm{N}^{\mathrm{o}}}$} & \multirow{2}{*}{$\frac{\text { artificial }}{\%}$} \\
\hline & No & $\%$ & & \\
\hline $\begin{array}{l}\text { I Cabeza encima } \\
\text { del ala derecha }\end{array}$ & 3 & 0.82 & 13 & 3.15 \\
\hline $\begin{array}{l}\text { II Cabeza hacia la } \\
\text { izquierda }\end{array}$ & 2 & 0.55 & 60 & 14.53 \\
\hline $\begin{array}{l}\text { III Cabeza fuera } \\
\text { de la cámara de } \\
\text { aire }\end{array}$ & 2 & 0.55 & 46 & 11.14 \\
\hline $\begin{array}{l}\text { IV Pata encima } \\
\text { de la cabeza o } \\
\text { pico }\end{array}$ & 8 & 2.19 & 27 & 6.53 \\
\hline $\begin{array}{l}\text { V Cabeza en el } \\
\text { extremo agudo }\end{array}$ & 3 & 0.82 & 8 & 1.94 \\
\hline $\begin{array}{l}\text { VI Cabeza entre } \\
\text { los muslos }\end{array}$ & 16 & 4.38 & 16 & 3.87 \\
\hline Total & 34 & 9.31 & 170 & 41.16 \\
\hline
\end{tabular}

En cuanto a las malposiciones más frecuentes en la incubación artificial (Tabla 7) fueron: la de tipo II (Cabeza dirigida hacia el lado izquierdo), que se relaciona con temperatura fluctuante en las incubadoras y raciones alimenticias en reproductoras; la que ocupa el segundo lugar es la de tipo III (el embrión ha rotado completamente lejos de la cámara de aire), que junto con la anterior (tipo II) son ocasionadas por la mala posición del huevo durante la incubación que es la causa más probable y que seguramente se debe a que la máquina se carga en forma accidental.

En lo referente a la deficiencia de cobalamina (Vitamina $\mathrm{B}_{12}$ ) y ácidos grasos esenciales (causa malposición I), como causales, son prácticamente nulas, debido al uso de una dieta balanceada.
La Tabla 8 presenta el resumen del número y porcentaje de las causas de mortalidad embrionaria, éstas de una manera general guardan relación con la contaminación de los huevos lo que provoca explosión de los mismos; también con los casos de malposiciones y malformaciones embrionarias observadas en el experimento; siendo estos casos las causas más frecuentes de mortalidad embrionaria. Las malformaciones embrionarias que predominaron fueron: hernia encefálica, procesos de hemorragia generalizada, dedos torcidos, abdomen abultado y embrión edematoso, en las dos clases de incubación. La hernia encefálica es causada como sostiene San Gabriel (1968), por factores genéticos y además por un tiempo prolongado de almacenamiento de los huevos a incubar y relacionados a problemas de alta temperatura en la incubadora eliminándose esta última para los resultados del presente estudio por cuanto la anomalía se presentó en un porcentaje muy pequeño, quedando los dos primeros motivos como causa del problema. La hemorragia generalizada es una malformación congénita y es causada como lo refiere San Gabriel (1968) por cáscara contaminada, también puede deberse a un exceso de formol en la fumigación, a la presencia de micotoxinas en el alimento de las reproductoras y de ciertos virus como el causante de la anemia infecciosa. En el presente estudio, puede deberse a un pequeño margen de contaminación que tiene que ser superado a través de programas eficientes de recojo de huevos limpios y desinfección de los mismos.

La presencia de ojos rudimentarios, cuyas causas según San Gabriel (1968) serían genéticas así como también atribuidas a temperatura de incubación fluctuante alta, factor éste último que quedaría excluido por la pequeña cantidad de estos casos.

Entonces se podría concluir en tres importantes motivos para la presentación de estas malformaciones con mayor frecuencia, características genéticas, almacenamiento prolongado y contaminación de la cáscara de los huevos. Comparativamente en la incubación artificial se encontró mayor porcentaje de embriones malformados y fue también el tipo de incubación donde se encontró un mayor número de huevos contaminados y además el almacenamiento prolongado contribuyó a la presencia de malformaciones embrionarias. 
Tabla 8. Malformaciones embrionarias (\%) en ambas clases de incubación.

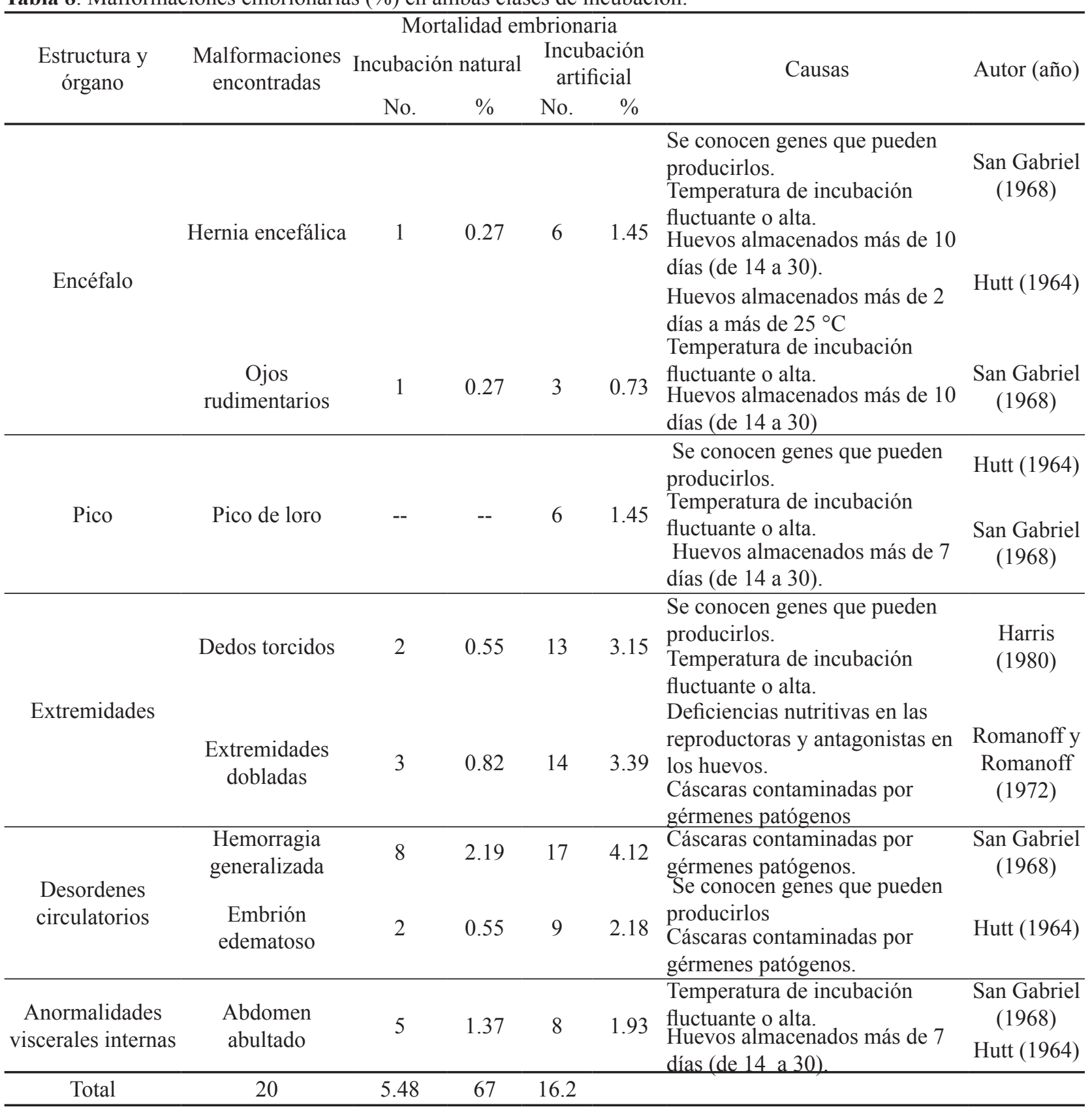

\section{Conclusiones}

Bajo las condiciones en las que se ha llevado a cabo el presente estudio, los huevos con almacenamiento de 3 semanas tuvieron menor mortalidad embrionaria en la incubación natural comparado con la incubación artificial. La incubación artificial tiene significativamente mayor porcentaje de malposiciones embrionarias. Las malposiciones más frecuentes en la incubación natural fueron: la malposición IV (pata sobre la cabeza) y la malposición VI (cabeza entre las patas). En la incubación artificial las malposiciones más frecuentes fueron: la del tipo II (cabeza hacia la izquierda) y la de tipo III (cabeza fuera de la cámara de aire). Hubo mayor porcentaje de malformación embrionaria en la incubación artificial, y las más frecuentes en ambos métodos de incubación fueron: procesos de hemorragia generalizada y abdomen abultado.

\section{Literatura citada}

Alda, R. 2003. Embrio-diagnóstico. In: Ed. Macari, M., Gonzales, E. Manejo da incubacao. Campinas: Facta, pp 499-514.

Blas M. 1978. Influencia del pulido de la cáscara en la incubabilidad de los huevos de pata criolla (Cairina moschata doméstica L.). Tesis Ingeniero Zootecnista. UNALM. Lima, Perú. 
Candela, H. 1975. Observaciones preliminares de la influencia de algunos factores en la incubación natural de los huevos de pata criolla (Cairina moschata doméstica L.). Tesis Ingeniero Zootecnista. UNALM. Lima, Perú.

Christensen, V., Donalson, W. y MC MURTRY, J. 1996. Physiological differences in late embryos from turkey breeders at different ages. Poultry Science 75: 172-178.

Christensen, V. 2001. Factors associated with early embryonic mortality. World's Poultry Science Journal 4 (57): 359-372.

Gonzales, R. 1976. Comparación de las pérdidas de peso durante la incubación natural y artificial en huevos de gallina (Gallus gallus) y de pata criolla (Cairina moschata doméstica L.). Tesis UNALM Lima-Perú.

Hamidu, J., Rieger, A., Fasenko, G. y Barreda, D. 2010. Dissociation of chicken blastoderm for examination of apoptosis and necrosis by flow cytometry. Poultry Science. Vol 89: 901- 909.

Harris, J. 1980. Manual de incubación. Extension Poultry Science. North Carolina State University

Harum, M., Veeneklaas, R., Visser, G. y Van Kamppen, M. 2001. Artificial Incubation of Muscovy Duck eggs: Why some eggs hatch and others do not. Poultry Science 80: 219-224.

Hutt, P. 1964. Animal Genetics. Ronald Press Company. New York.

Kalita, N., Pathak, N., Ahmed, M. y Saikia, G. 2013. Various causes related to dead-in- shell embryos of crossbred (PB-2 x Indigenous) chicken egg. Veterinary World 6(10): 774-777.

Landauer, W., 1967. The Hatchability of chicken Egg as Influenced by Environment and Heredity. Agricultural Experiment Station. Bulletins 215, 236 y 262 USA.

Narbaiza, I. 2005. Aumente la Producción del Pato Real ( $\underline{\text { Cairina }} \underline{\text { moschata)}}$. Puerto Ayacucho, Estado de Amazonas, Venezuela.

Pacheco, V. 1976. Influencia del medio de almacenamiento en la incubabilidad de huevos de pata criolla (Cairina moschata doméstica L.) bajo condiciones naturales. Tesis Ingeniero Zootecnista. UNALM. Lima, Perú.

Reinhart, B. y J. Hurnik. 1976. The effect of temperature and storage time during pre-incubation period. Poultry Science 55: 1631-1640.

Reis, L., Gama, L., Chaveiro, T y Soares, M. 1997. Effects of short storage conditions and broiler breeder age on hatchability, hatching time and chick weights. Poultry Science Vol. 76 (11) : 1457-1466.

Romanoff, A. y Romanoff, J. 1972. Pathogenesis of the avian embryo an analysis of causes of malformations and prenatal deanth. Wiley. Interscience. New York

Rivas, P. 1981. Influencia del enfriamiento y de la posición del huevo en la incubadora artificial de los huevos de patas criollas (Cairina moschata doméstica L Tesis Ingeniero Zootecnista. UNALM. Lima, Perú.
San Gabriel, A. 1968. Patología de la incubación y enfermedades del polluelo. Editorial AEDOS. Barcelona.

Vargas, J. 1985. Efecto de la Temperatura Inicial de incubación sobre la incubabilidad artificial de los huevos

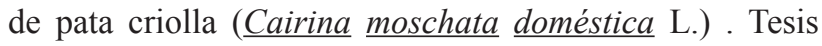
Ingeniero Zootecnista. UNALM. Lima, Perú.

Vela, C. 1982. Incubación natural, artificial y mixta de los huevos de patas criollas (Cairina moschata doméstica L.). Tesis Ingeniero Zootecnista. UNALM. Lima, Perú.

Vilchez, F. 1975. Ensayos de diferentes humedades relativas en la incubación artificial de los huevos de patas criollas (Cairina moschata doméstica L.). Tesis Ingeniero Zootecnista. UNALM. Lima, Perú. 